\title{
Vitamin D gegen diabetische Neuropathie?
}

\author{
Die hoch dosierte, einmalige, intramuskuläre Gabe von Vitamin D führte in einer Beobachtungs- \\ studie zu einer deutlichen Besserung der schmerzhaften diabetischen Neuropathie.
}

_ Etwa jeder fünfte Diabetiker leidet an einer Neuropathie mit symmetrischen Parästhesien, Dysästhesien, Schmerzen und Berührungsempfindlichkeit. Eine zuverlässige Therapie gibt es nicht. Allerdings gibt es Hinweise auf eine Korrelation des Symptomauftretens mit dem Vitamin-D-Spiegel.

Für eine Beobachtungsstudie wurde nun 143 Diabetikern (vorwiegend Typ 2) mit schwerer schmerzhafter Neuropathie einmalig eine hohe Dosis von 600.000 IE Vitamin D intramuskulär in jiziert. Mit drei verschiedenen Scores wurden zuvor sowie viermal danach Stärke und Qualität der Schmerzen be- stimmt. Im Labor wurden diverse Parameter einschließlich der Vitamin-DSpiegel vor und nach der Injektion bestimmt.

Nach 20 Wochen hatten Stärke und Qualität der Schmerzen erheblich und signifikant abgenommen ( $\mathrm{p}<0,0001)$. Die Besserung war bereits nach acht Wochen erkennbar und verstärkte sich danach noch. Die Vitamin-D-Spiegel stiegen von 31,7 auf 46,2 ng/ml, wobei allerdings die Besserung bei allen Ausgangswerten gleich stark ausfiel.

- Basit A, Basit KA, Fawwad A et al. Vitamin D for the treatment of painful diabetic neuropathy. BMJ Open Diab Res Care. 2016;4:e000148.

\section{KOMMENTAR}

Die Besserung ist bemerkenswert, zumal sie mit den Vitamin-D-Ausgangswerten nicht korreliert - also die Studienhypothese nicht bestätigt. Die Autoren konzedieren, dass es sich nur um eine Beobachtungstudie handelt. Allerdings erwartet man von einem Placeboeffekt eine schnelle, kurzlebige Wirkung, nicht aber eine allmähliche, konstante Verstärkung bis zu einer Dauer von 20 Wochen. Die Vitamin-D-Gabe könnte deutlich attraktiver sein als die in den Leitlinien empfohlenen Maßnahmen bei diabetischer Neuropathie. Eine kontrollierte Studie über längere Dauer ist wünschenswert.

Prof. Dr. med. H. Holzgreve

\section{Hier steht eine Anzeige.}

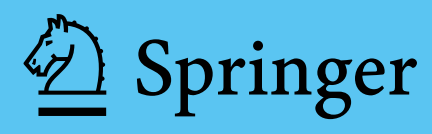

